\title{
NONCOMPACT SIMPLICES
}

\author{
BY \\ S. SIMONS( $\left.{ }^{1}\right)$
}

\begin{abstract}
A bounded, but not necessarily closed, (Choquet) simplex in $R^{n}$ with nonempty interior is the intersection of $n+1$ half-spaces. There is no bounded simplex with nonempty interior in an infinite dimensional Hausdorff real linear topological space.
\end{abstract}

0. Introduction. We suppose that $X$ is a nonzero real linear space. We use the symbols $t, u, v, w, x, y, z$ to represent elements of $X$ and $\alpha, \beta, \gamma, \delta, \eta$ to denote real numbers $\geqq 0$. We say that $S(\subset X)$ is a simplex if $S$ is nonempty and convex and whenever $(x+\alpha S) \cap(y+\beta S) \neq \varnothing$ then there exist $z, \gamma$ such that $(x+\alpha S) \cap(y+\beta S)$ $=z+\gamma S$. The main results of this paper are: a bounded simplex in $R^{n}$ with nonempty interior is the intersection of $n+1$ half-spaces (each of which can be open or closed) and there is no bounded simplex with nonempty interior in an infinite dimensional Hausdorff linear topological space. These results are in $\$ \$ 2$ and 3 , respectively. In $§ 1$, we discuss a nontopological boundedness condition.

1. The closure of a simplex is sometimes a simplex.

1. Definition. If $\varnothing \neq A \subset X$ and $x \in X \backslash\{0\}$ we write

$$
D(A, x)=\sup \{\alpha: \alpha x \in A-A\} \text {. }
$$

$(D(A, x)$ is the "diameter of $A$ in the direction of $x$ ".)

2. Lemma. Let $X$ be a linear topological space, $S(\subset X)$ be a simplex and $z \in$ int $S$. If $t, t^{\prime}, u, u^{\prime} \in \bar{S}$ are such that $t^{\prime}-t=u^{\prime}-u \neq 0$ and $\phi$ is a nonzero continuous linear functional on $X$ such that $\phi(t)=\inf \phi(S), \phi(u)=\sup \phi(S)$ then $D\left(S, t^{\prime}-t\right)=\infty$.

Proof. We suppose $0<\alpha<\frac{1}{2}$. Since $t, u \in \bar{S}$ and $z \in$ int $S$,

$$
(1-\alpha) t+\alpha z \in S
$$

and

$$
\alpha t+(1-2 \alpha) u+\alpha z \in S
$$

from which

$$
(1-\alpha) t+\alpha z \in(1-2 \alpha)(t-u)+S
$$

Received by the editors May 22, 1969 and, in revised form, September 26, 1969.

AMS Subject Classifications. Primary 5230, 4601, 4606.

Key Words and Phrases. Choquet simplex, linearly compact, abstract $L$-space.

( ${ }^{1}$ This research was supported in part by National Science Foundation Grant GP-8394.

Copyright (C) 1970, American Mathematical Society 
hence there exist $w, \beta$ such that

$$
S \cap[(1-2 \alpha)(t-u)+S]=w+\beta S .
$$

Now

hence

$$
S \supset w+\beta S \subset(1-2 \alpha)(t-u)+S
$$

from which

$$
\begin{aligned}
\inf \phi(S) & \leqq \phi(w)+\beta \inf \phi(S) \leqq \phi(w)+\beta \sup \phi(S) \\
& \leqq(1-2 \alpha) \phi(t-u)+\sup \phi(S)
\end{aligned}
$$

$$
\beta \operatorname{diam} \phi(S) \leqq(1-2 \alpha)[\phi(t)-\phi(u)]+\operatorname{diam} \phi(S)=2 \alpha \operatorname{diam} \phi(S) .
$$

Since int $S \neq \varnothing, \operatorname{diam} \phi(S)>0$ and so

$$
\beta \leqq 2 \alpha .
$$

From (1), (2) and (3)

$$
(1-\alpha) t+\alpha z \in w+\beta S
$$

Now $\phi\left(t^{\prime}\right) \geqq \inf \phi(S)=\phi(t)$ and so $\phi\left(t^{\prime}-t\right) \geqq 0$. Similarly $\phi\left(u^{\prime}-u\right) \leqq 0$. Hence, since $t^{\prime}-t=u^{\prime}-u, \phi\left(t^{\prime}-t\right)=\phi\left(u^{\prime}-u\right)=0$ and so $\phi\left(t^{\prime}\right)=\inf \phi(S)$ and $\phi\left(u^{\prime}\right)=\sup \phi(S)$. Further, $t^{\prime}-u^{\prime}=t-u$ and so, by an argument similar to that above,

$$
(1-\alpha) t^{\prime}+\alpha z \in w+\beta S \text {. }
$$

Hence $\beta D\left(S, t^{\prime}-t\right) \geqq 1-\alpha$ and so, from (4), $2 \alpha D\left(S, t^{\prime}-t\right) \geqq 1-\alpha$. Letting $\alpha \rightarrow 0$ gives the required result.

3. Definition. If $\varnothing \neq A \subset X$ we say that $A$ is $D$-bounded if, for all $x \in X \backslash\{0\}$, $D(A, x)<\infty$.

4. THEOREM. If $X$ is a linear topological space, $S(\subset X)$ is a D-bounded simplex and int $S \neq \varnothing$ then $\bar{S}$ is a simplex.

Proof. It is immediate that int $S$ is a $D$-bounded simplex. Further $\bar{S}=\overline{\operatorname{int} S}$ so we can suppose, without loss of generality, that $S$ is open. Suppose $T=(x+\alpha \bar{S})$ $\cap(y+\beta \bar{S}) \neq \varnothing$. If $T=\{z\}$ then $T=z+0 \bar{S}$ and the result follows. So we suppose that $T \ni v, T \ni w$ where $v \neq w$. This implies that $\alpha>0, \beta>0$. We shall show that

$$
(x+\alpha S) \cap(y+\beta S) \neq \varnothing .
$$

Indeed, if (5) is false then there exists a nonzero continuous linear functional $\phi$ on $X$ such that

$$
\inf \phi(x+\alpha S)=\phi(v)=\sup \phi(y+\beta S) .
$$

We suppose, without loss of generality, that $\beta \leqq \alpha$. Then, from Lemma 2 with

$$
t=\frac{v-x}{\alpha}, \quad u=\frac{v-y}{\beta}, \quad t^{\prime}=\frac{w-x}{\alpha}, \quad u^{\prime}=\frac{1}{\beta}\left\{\left(1-\frac{\beta}{\alpha}\right) v+\frac{\beta}{\alpha} w-y\right\},
$$


$D\left(S, t^{\prime}-t\right)=\infty$ contradicting our hypothesis that $S$ is $D$-bounded. Hence (5) is true. Since $S$ is a simplex, there exist $z, \gamma$ such that

$$
(x+\alpha S) \cap(y+\beta S)=z+\gamma S .
$$

It follows by considering gauge functionals that if $A, B$ are convex and open in $X$ and $A \cap B \neq \varnothing$ then $\overline{A \cap B}=\bar{A} \cap \bar{B}$. Hence from (6),

$$
(x+\alpha \bar{S}) \cap(y+\beta \bar{S})=z+\gamma \bar{S} .
$$

Thus $\bar{S}$ is a simplex.

5. RemarK. Let $X=R^{2}$ and $S=(0,1) \times R$. Then $S$ is a simplex but $\bar{S}$ is not a simplex. This example, suggested by $D$. Randtke, shows that some boundedness condition on $S$ is essential in Theorem 4 . In the next theorem we explore the properties of $D$-boundedness.

6. THEOREM. (a) If $X$ is a Hausdorff linear topological space, $\varnothing \neq A \subset X$ and $A$ is bounded then $A$ is $D$-bounded.

(b) If $\varnothing \neq A \subset R^{n}$ and $A$ is convex and $D$-bounded then $A$ is bounded.

Proof. (a) Let $x \in X \backslash\{0\}, U$ be a balanced neighborhood of 0 such that $U \not x$ and $V$ be a neighborhood of 0 such that $V-V \subset U$. If $\alpha x \in V-V$ then $\alpha x \in U$ and so $\alpha<1$. Hence $D(V, x) \leqq 1$. But $A$ is absorbed by $V$ hence $D(A, x)<\infty$.

(b) We first translate $A$ to contain 0 and then replace $R^{n}$ by the subspace spanned by $A$. We may, therefore, suppose that int $A \ni z$, say. If $A$ is unbounded then, from the argument used in [2, p. 370], $\bar{A}$ contains an infinite half-line $l$. Then $A \supset \frac{1}{2}(z+l)$ and so $A$ is not $D$-bounded.

7. Remark. We give an example of a $D$-bounded open simplex in $C[0,1]$. Let

$$
S=\left\{x: x \in C[0,1], x(t)>0 \text { for all } t \in[0,1], \int x<1\right\} .
$$

$S$ is clearly open. $S$ is $D$-bounded, for if $x \in C[0,1] \mid\{0\}$ and $y, y+\alpha x \in S$ then

$$
0 \leqq y+\alpha x
$$

and

$$
\int(y+\alpha x) \leqq 1
$$

if, for some $t \in[0,1], x(t)<0$ then, from (7), $\alpha|x(t)| \leqq y(t)$ hence $D(S, x)<\infty$; if, on the other hand, for all $t \in[0,1], x(t) \geqq 0$ then $\int x>0$ and, from (8), $\alpha \int x \leqq 1-\int y$ and, again, $D(S, x)<\infty$. Finally, $S$ is a simplex, for if $(x+\alpha S)$ $\cap(y+\beta S) \neq \varnothing$ then

$$
(x+\alpha S) \cap(y+\beta S)=x \vee y+\left[\left(\int x+\alpha\right) \wedge\left(\int y+\beta\right)-\int x \vee y\right] S .
$$

We observe that $S$ is unbounded. We shall see in Theorem 14 that this is, in fact, forced by the other conditions on $S$. 
The above example emerged from a conversation with $\mathbf{M}$. Rosenfeld.

2. Simplices in $R^{n}$. We suppose throughout this section that $X=R^{n}$. If $S(\subset X)$ is a bounded simplex then, by using the argument sketched in the proof of Theorem 6 (b), we can suppose that int $S \neq \varnothing$ and, to avoid trivial cases, that $n>1$.

If $A \subset X$ we write conv $A$ for the convex hull of $A$. If $A=\left\{x_{1}, \ldots, x_{k}\right\}$ is a finite set we write

$$
\operatorname{conv}_{+} A=\left\{\sum_{i=1}^{k} \alpha_{i} x_{i}: \alpha_{i}>0, \sum_{i=1}^{k} \alpha_{i}=1\right\} .
$$

If $S$ is as above then, from Theorem 6 (a) and Theorem $4, \bar{S}$ is a compact simplex hence [1, Remarks following Definition 5] and [3, Proposition 9.11, p. 75] there exists affinely independent $v_{0}, \ldots, v_{n} \in X$ such that $\bar{S}=\operatorname{conv}\left\{v_{0}, \ldots, v_{n}\right\}$. (We shall return to this result in Theorem 12.) Since $S$ is convex, $S \supset \operatorname{conv}_{+}\left\{v_{0}, \ldots, v_{n}\right\}$.

8. Lemma. We suppose that $X, S, v_{0}, \ldots, v_{n}$ are as above and, further, that $v_{0}=0$. Then $v_{1}, \ldots, v_{n}$ form a basis of $X$. We write $\psi_{1}, \ldots, \psi_{n}$ for the dual basis of $X$ and $\psi$ for $\psi_{1}+\cdots+\psi_{n}$. We define the lattice ordering $\leqq$ (with lattice operations $\vee$ and $\wedge$ ) on $X$ by " $x \leqq y$ " means that "for all $i=1, \ldots, n, \psi_{i}(x) \leqq \psi_{i}(y)$ ". We write $w=n^{-1}\left(v_{1}+\cdots+v_{n}\right) \in \bar{S}$.

(a) If $x+\alpha S \supset z+\delta S$ then $z \geqq x$ and $\psi(z)+\delta \leqq \psi(x)+\alpha$.

(b) If $\eta>0, z \geqq x$ and $\psi(z)+\eta<\psi(x)+\alpha$ then $z+\eta w \in x+\alpha S$.

(c) If $(x+\alpha S) \cap(y+\beta S)=T \neq \varnothing$ then $T=x \vee y+\gamma S$, where

$$
\gamma=\min \{\psi(x)-\psi(x \vee y)+\alpha, \psi(y)-\psi(x \vee y)+\beta\} \text {. }
$$

(d) If $y \in S, z \geqq 0, \psi(y)=\psi(z)=1$ and

$$
\left\{i: 1 \leqq i \leqq n, \psi_{i}(z)=0\right\} \subset\left\{i: 1 \leqq i \leqq n, \psi_{i}(y)=0\right\}
$$

then $z \in S$.

(e) If $x \in \alpha S$ and $y \in \beta S$ then $x \wedge y \in \gamma S$, where

$$
\gamma=\min \{\psi(x \wedge y)-\psi(x)+\alpha, \psi(x \wedge \dot{y})-\psi(y)+\beta\} .
$$

Proofs. (a) follows by taking the images under $\psi_{i}$ and $\psi$ and the inf and sup, respectively. In (b) the conditions imply that $z-x+\eta w \in \alpha$ int $S \subset \alpha S$.

(c) Since $S$ is a simplex; there exist $z, \delta$ such that $T=z+\delta S$. From (a),

$$
z \geqq x, \quad z \geqq y, \quad \psi(z)+\delta \leqq \psi(x)+\alpha, \quad \psi(z)+\delta \leqq \psi(y)+\beta
$$

and so $z \geqq x \vee y$. If we had $z \neq x \vee y$ then, for some $i$ such that $1 \leqq i \leqq n$ and for some $\eta>0, \psi_{i}(x \vee y)+\eta<\psi_{i}(z)$ hence $\psi(x \vee y)+\eta<\psi(z)$. From (9),

$$
\psi(z) \leqq \min \{\psi(x)+\alpha, \psi(y)+\beta\}
$$

thus, from (b), $x \vee y+\eta w \in(x+\alpha S) \cap(y+\beta S)=T$ hence $x \vee y+\eta w \geqq z$. Letting $\eta \rightarrow 0$ gives $x \vee y \geqq z$, hence $z=x \vee y$, as required. Returning to (9) we now see that $0 \leqq \delta \leqq \gamma$. If $\gamma=0$ then clearly $\gamma=\delta$. If $\gamma>0$ then, for any $\eta$ such that $0<\eta<\gamma$, 
$\psi(x \vee y)+\eta<\min \{\psi(x)+\alpha, \psi(y)+\beta\}$ and so, as above, $x \vee y+\eta w \in z+\delta S=x \vee y$ $+\delta S$. Hence $\eta w \in \delta S$ which implies that $\eta \leqq \delta$. Letting $\eta \rightarrow \gamma$ gives that $\gamma \leqq \delta$. Hence $\gamma=\delta$, as required.

(d) If $\alpha>0$ is sufficiently small then $z-\alpha y \geqq 0$ and $z-\alpha y+\alpha$ int $S \subset$ int $S$ hence $(z-\alpha y+\alpha S) \cap S \neq \varnothing$. From (c), $(z-\alpha y+\alpha S) \cap S=(z-\alpha y+\alpha S)$, hence $z-\alpha y$ $+\alpha S \subset S$. In particular, $z=z-\alpha y+\alpha y \in S$.

(e) We have $0 \in(-x+\alpha S) \cap(-y+\beta S)$. The result is immediate from (c).

9. Lemma. We suppose that $X, S, v_{0}, \ldots, v_{n}$ are as in the discussion preceding Lemma 8. We write $\mathcal{N}=\{F: \varnothing=F \subset\{0, \ldots, n\}\}$ and, if $F \in \mathscr{N}$,

$$
[F]=\operatorname{conv}_{+}\left\{v_{i}: i \in F\right\} .
$$

Also we write $\mathscr{F}=\{F: F \in \mathscr{N},[F] \cap S \neq \varnothing\}$.

(a) $S=\bigcup\{[F]: F \in \mathscr{F}\}$.

(b) $G \in \mathscr{F}, F \in \mathscr{N}$ and $G \subset F$ imply $F \in \mathscr{F}$.

(c) If $F, G \in \mathscr{F}$ and $F \cap G \neq \varnothing$ then $F \cap G \in \mathscr{F}$.

Proofs. (a) We first observe that $S \subset \bar{S}=\bigcup\{[F]: F \in \mathscr{N}\}$ hence

$$
S \subset \bigcup\{[F]: F \in \mathscr{F}\} .
$$

Now we suppose $F \in \mathscr{F}$. If $F=\{0, \ldots, n\}$ then $[F]=$ int $S \subset S$. If $F \varsubsetneqq\{0, \ldots, n\}$ we assume, without loss of generality, that $F \ngtr 0$ and we translate $S$ so that $v_{0}=0$. There exists $y$, say, such that $y \in[F] \cap S$. It follows from Lemma 8 (d) that, if $z \in[F]$ then $z \in S$, i.e. $[F] \subset S$. Hence $\bigcup\{[F]: F \in \mathscr{N}\} \subset S$.

(b) As in (a) we suppose, without loss of generality, that $F \nexists 0, v_{0}=0$ and $y \in[G] \cap S$. From Lemma 8 (d) again, $[F] \subset S$, hence $F \in \mathscr{F}$.

(c) This time we reduce the problem to the case $F \cap G \ni 0$ and $v_{0}=0$. We write $x=\sum\left\{v_{i}: i \in F\right\}$ and $y=\sum\left\{v_{i}: i \in G\right\}$. Then, from (a), $x \in(\psi(x)+1) S$ and $y \in(\psi(y)+1) S$. From Lemma $8(\mathrm{e}), x \wedge y \in(\psi(x \wedge y)+1) S$. But

$$
x \wedge y=\sum\left\{v_{i}: i \in F \cap G\right\}
$$

hence $F \cap G \in \mathscr{F}$.

10. THEOREM. If $S$ is a bounded simplex in $R^{n}$ and int $S \neq \varnothing$ then $S$ is the intersection of $n+1$ half-spaces.

Proof. We use the notation of Lemma 9. If there exists $F, G \in \mathscr{F}$ such that $F \cap G=\varnothing$ then, using (b) and (c) of Lemma 9, for each $i \in\{0, \ldots, n\},\{i\}=$ $(F \cup\{i\}) \cap(G \cup\{i\})$ hence $\{i\} \in \mathscr{F}$ and so $\mathscr{F}=\mathscr{N}$. Hence, from Lemma 9 (a), $S=\bar{S}$ which is the intersection of $n+1$ closed half-spaces.

If, on the other hand, for all $F, G \in \mathscr{F}, F \cap G \neq \varnothing$ then, from (b) and (c) of Lemma 9 again, $\mathscr{F}$ is a filter of subsets of $\{0, \ldots, n\}$ and so there exists $F_{0} \in \mathscr{N}$ such that $\mathscr{F}=\left\{F: F \in \mathscr{N}, F \supset F_{0}\right\}$. From Lemma 9 (a)

$S=\left\{\lambda_{0} v_{0}+\cdots+\lambda_{n} v_{n}: \lambda_{i}>0\right.$ for all $i \in F_{0}$,

$$
\left.\lambda_{i} \geqq 0 \text { for all } i \in\{0, \ldots, n\} \backslash F_{0}, \lambda_{0}+\cdots+\lambda_{n}=1\right\},
$$


which is the intersection of $m$ open and $n+1-m$ closed half-spaces, where $m$ is the cardinality of $F_{0}$.

11. REMARKS. We leave to the reader the proof of the converse of Theorem 10 , that any bounded set with nonempty interior that is the intersection of $n+1$ halfspaces is a simplex. We observe that there are exactly $n+2$ affinely different bounded simplices with nonempty interior in $R^{n}$, distinguished by the minimum dimension of an "open face" (i.e., a set $[F]$ for $F \in \mathscr{N}$ ). On the other hand there are just 3 topologically different such simplices. Finally, any such simplex contains exactly 0 , 1 or $n+1$ of its vertices.

3. Bounded simplices with nonempty interior. In this section we return to the general notation of $\S 1$.

12. TheOREM. If $S$ is a linearly compact (see [2]) simplex in $X, x \in S, \eta>0$ and $x-\eta(S-x) \subset S$, then $S$ is the convex hull of a finite affinely independent set containing at most $1+1 / \eta$ points.

Proof. Let $C$ be the cone $\{(\alpha y, \alpha): \alpha \geqq 0, y \in S\}$ in $E \times R$. From [2], $C$ induces a lattice ordering on $L=C-C$. The map $\phi:(x, \alpha) \rightarrow \alpha$ is a positive linear functional on $L$, and $f \in L, f \geqq 0$ and $\phi(f)=0$ imply that $f=0$. If $f \in L$ we write $\|f\|=\phi(|f|)$. $\|\cdot\|$ is a norm on $L$ with respect to which $L$ is a normed lattice and $\|\cdot\|$ is additive on the positive elements of $L$. Hence the completion, $\tilde{L}$, of $L$ is an abstract $L$-space.

We write $g=(1+1 / \eta)(x, 1)$. If $f \in L, f \geqq 0$ then there exists $\alpha \geqq 0, y \in S$ such that $f=(\alpha y, \alpha)$. Then

$$
\begin{aligned}
\phi(f) g-f & =\alpha(1+1 / \eta)(x, 1)-(\alpha y, \alpha) \\
& =(\alpha / \eta)(x-\eta(y-x), 1) \geqq 0
\end{aligned}
$$

by hypothesis, from which $g$ is an order unit for $\tilde{L}$. From $[4$, V. 8.6, Corollary 1 , p. 249], $L$ is finite dimensional and hence, for some $n \geqq 1, L=\tilde{L} \cong l_{n}^{1}$.

We suppose that $f_{1}, \ldots, f_{n}$ are the elements of $L$ that correspond to the basic unit vectors of $l_{n}^{1}$. For each $i=1, \ldots, n$ there exists $x_{i} \in S$ such that $f_{i}=\left(x_{i}, 1\right)$. It follows from the linear independence of $\left\{f_{i}\right\}$ that $\left\{x_{i}\right\}$ are affinely independent and from $L=\operatorname{lin}\left\{f_{i}\right\}$ that $S=\operatorname{conv}\left\{x_{i}\right\}$.

There exist $\alpha_{1}, \ldots, \alpha_{n} \geqq 0, \sum \alpha_{i}=1$ such that $(x, 1)=\sum \alpha_{i} f_{i}$. We suppose that $i \in\{1, \ldots, n\}$ has been chosen so that $\alpha_{i} \leqq 1 / k$. From (10), $f_{i} \leqq \phi\left(f_{i}\right) g=\left\|f_{i}\right\| g=g$ $=(1+1 / \eta) \sum \alpha_{i} f_{i}$ hence, projecting along the vector $f_{i}, 1 \leqq(1+1 / \eta) \alpha_{i} \leqq(1+1 / \eta) 1 / k$. Thus $k \leqq 1+1 / \eta$, as required.

13. Remarks. The constant $1+1 / \eta$ above is the best possible in the sense that if $S$ is the convex hull of $n+1$ affinely independent points and $x$ is the barycenter of $S$ then $x-n^{-1}(S-x) \subset S$. This observation is due to $\mathrm{D}$. Randtke. Theorem 12 generalizes the results used at the beginning of $\S 2$.

14. THEOREM. There is no bounded simplex with nonempty interior in an infinite dimensional Hausdorff linear topological space. 
Proof. If $X$ is a Hausdorff linear topological space and $S(\subset X)$ is a bounded simplex with $x \in$ int $S$ then, from Theorem 6 (a) and Theorem $4, \bar{S}$ is a simplex. $\bar{S}$ is linearly compact and, for some $\eta>0$, satisfies the condition of Theorem 12 . It now follows from Theorem 12 that $\operatorname{dim} X<\infty$.

15. Remark. The above result should be compared with Remark 7.

\section{REFERENCES}

1. G. Choquet, Existence et unicité des représentations intégrales au moyen des points extrémaux dans les cônes convexes, Séminaire Bourbaki, Exposés 139, Secrétariat mathématique, Paris, 1959, 15 pp. MR 28 \#1090.

2. D. G. Kendall, Simplexes and vector lattices, J. London Math. Soc. 37 (1962), 365-371. MR 25 \#2423.

3. R. R. Phelps, Lectures on Choquet's theorem, Van Nostrand, Princeton, N. J., 1966. MR 33 \#1690.

4. H. H. Schaefer, Topological vector spaces, Macmillan, New York, 1966. MR 33 \#1689.

UNIVERSITY OF CALIFORNIA,

Santa Barbara, California 93106 